\title{
Structural and Electronic Properties of Gold Clusters
}

\author{
Denitsa Alamanova, ${ }^{1}$ Yi Dong, ${ }^{2}$ Habib ur Rehman, ${ }^{3}$ \\ Michael Springborg, ${ }^{4}$ and Valeri G. Grigoryan ${ }^{5}$ \\ Physical and Theoretical Chemistry, \\ University of Saarland, \\ 66123 Saarbrücken, \\ Germany
}

Received 30 June, 2005; accepted in revised form 30 June, 2005

\begin{abstract}
We study the structure and energetics of $\mathrm{Au}_{N}$ clusters by means of parameterfree density-functional calculations $(N \leq 8)$, jellium calculations $(N \leq 60)$, embeddedatom calculations $(N \leq 150)$, and parameterized density-functional calculations $(N \leq 40)$ in combination with different methods for determining the structure of the lowest total energy. By comparing the results from the different approaches, effects due to geometric packing and those due to the electronic orbitals can be identified. Different descriptors that highlight the results of the analysis are presented and used.
\end{abstract}

Keywords: Gold clusters, structure, stability, density-functional calculations, embeddedatom calculations

PACS: 36.40.-c, 36.90.+f, 61.46.+w, 73.22.-f

\section{Introduction}

Clusters of gold atoms have become the maybe mostly studied class of clusters [1], partly due to the possibility to apply them in electronic devices [2], nanomaterials [3] and catalysis [4. Despite this popularity only little consensus has been reached concerning the structure of these clusters. Many studies devoted to this issue use combinations of experimental and theoretical methods [5, 6, 7].

From a theoretical point of view, gold clusters offer an additional challenge due to the importance of relativistic effects, most notably the strong spin-orbit couplings. Thus, in parameter-free, electronic-structure calculations, one has to use special, relativistic potentials. With those, the smallest gold clusters are found to be planar [5, 8, 9, 10, 11, 12, 13, whereas their exclusion leads to three-dimensional structures [14, 15].

However, for not-too-small gold clusters, it becomes increasingly difficult to apply parameterfree, electronic-structure methods in the calculation of the properties of the gold clusters, partly because the computational demands scale with the size of the system to at least the third power, and partly because the number of metastable structures grows very rapidly with cluster size. Thus, in parameter-free studies one often has to make significant assumptions on the structure of the system,

${ }^{1}$ e-mail: deni@springborg.pc.uni-sb.de

2e-mail: y.dong@mx.uni-saarland.de

${ }^{3}$ e-mail: haur001@rz.uni-saarland.de

${ }^{4}$ Corresponding author. e-mail: m.springborg@mx.uni-saarland.de

${ }^{5}$ e-mail: vg.grigoryan@mx.uni-saarland.de 
as for instance is the case in the study of Häberlen et al. [16]. One of the greatest disadvantages of the first-principles methods is their incapability of optimizing large number of randomly generated initial structures and thereby determining the true global total-energy minimum. One example of this is provided by $\mathrm{Au}_{7}$ and $\mathrm{Au}_{8}$ for which the structure was predicted in 2000 by Häkkinen and Landman [10 to be a planar structure with $\mathrm{D}_{2 h}$ symmetry and a three-dimensional capped tetrahedron, respectively. Three years later, Häkkinen et al. [5] showed that the lowest-totalenergy structure of $\mathrm{Au}_{7}^{-}$corresponds to a planar structure consisting of a rhombus, capped with an additional atom on three of its sides, and that the ground state of $\mathrm{Au}_{8}^{-}$is the same rhombus, with its 4 sides capped. In 2005, the results for $\mathrm{Au}_{8}$ were confirmed by Walker [12] and Remacle et al. 13], whereas both works found a planar capped hexagon to be the global minimum of neutral $\mathrm{Au}_{7}$.

Approximate methods may provide a useful alternative to the parameter-free methods. They are computationally less demanding, thus allowing for a detailed search in structure space so that structures for clusters with well above 100 atoms can be predicted in an unbiased way. On the other hand, being approximate it is not obvious how reliable they are. It is the purpose of this work to address this issue. To this end, gold clusters provide an excellent playground, partly because of the large uncertainty concerning their structure in combination with the large amount of studies on these clusters, but also partly because for clusters both geometric packing effects and electronic shell effects may be responsible for the occurrence of certain particularly stable clusters (the so-called magic numbers). The approximate methods often make different approximations on the relative importance of these two effects.

One class of approximate methods is formed by the embedded-atom methods (EAM) that only indirectly includes electronic effects and, therefore, first of all (but not exclusively) put emphasis on packing effects. Both the EAM [17, 18, 19, the Sutton-Chen [14, the Murrell-Mottram [15], and the many-body Gupta potential [20, 21, 22, 23, 24, 25] (that all share the property of including electronic effects only very approximately) have all been applied in unbiased structure optimizations for gold clusters with up to 80 atoms.

One of the, maybe, surprising outcomes of these studies is that the results depend very sensitively on the applied method, i.e., on the (more or less) approximate description of the interatomic interactions and on the method for structure optimization, see, e.g., 26, 27]. One reason may be a subtle interplay between electronic and geometric effects, i.e., that the particularly stable structures of $\mathrm{Au}_{N}$ clusters are dictated partly by the closing of electronic shells and partly by geometric packing effects. Here, the various potentials give different relative importance to the two effects.

The purpose of this contribution is to discuss general methods for calculating the properties of clusters, using gold clusters as the prototype. In parallel we shall also discuss the special properties of the gold clusters, specifically, with special emphasis on the issue above, i.e., whether electronic or packing effects are important in dictating the particularly stable clusters, and, moreover, how the different more or less accurate methods perform in calculating the properties of the clusters.

\section{Methods}

\section{$2.1 \quad$ Total-energy methods}

The smallest gold clusters with up to eight atoms were treated with parameter-free electronicstructure calculations using the GAUSSIAN03 program package 28]. We performed density-functional (DFT) calculations using the generalized-gradient approximation (GGA) of Perdew, Burke, and Ernzerhof 29, 30]. These calculations treat, in principle, all types of interactions, i.e., electronic and geometric effects, at an exact level.

In addition we also performed self-consistent, electronic-structure calculations on spherical clusters where only the 11 ( $5 d$ and $6 s$ ) valence electrons per Au atom were treated explicitly, whereas 
all core electrons and the nuclei were smeared out to a uniform jellium background [31, 32. This model focuses essentially only on electronic effects.

As an alternative we also considered the embedded-atom method (EAM) in the parameterization of Voter and Chen [33, 34, 35. According to this method, the total energy for a system of $N$ atoms is written as

$$
\begin{aligned}
E_{\mathrm{tot}} & =\sum_{i} E_{i} \\
E_{i} & =F_{i}\left(\rho_{i}^{h}\right)+\frac{1}{2} \sum_{j(\neq i)} \phi_{i j}\left(r_{i j}\right) \\
\rho_{i}^{h} & =\sum_{j(\neq i)} \rho_{i}^{a}\left(r_{i j}\right),
\end{aligned}
$$

i.e., as a sum of atomic components, each being the sum of two terms. The first term is the energy that it costs to bring the atom of interest into the electron density provided by all other atoms, and the second term is a pair-potential term. Both terms are assumed depending only on the distances between the neighbouring atoms, and do therefore not include any directional dependence. Accordingly, the EAM emphasizes geometrical effects, whereas electronic effects are included only very indirectly.

Furthermore, we used the density-functional tight-binding method (DFTB) as developed by Seifert and coworkers 36, 37. With this method, the binding energy is written as the difference in the orbital energies of the compound minus those of the isolated atoms, i.e., as

$$
\sum_{i} \epsilon_{i}-\sum_{m} \sum_{i} \epsilon_{m i}
$$

(with $m$ being an atom index and $i$ an orbital index), augmented with pair potentials,

$$
\sum_{m_{1} \neq m_{2}} U_{m_{1}, m_{2}}\left(\left|\vec{R}_{m_{1}}-\vec{R}_{m_{2}}\right|\right)
$$

(with $\vec{R}_{m}$ being the position of the $m$ th atom). In calculating the orbital energies we need the Hamilton matrix elements $\left\langle\chi_{m_{1} n_{1}}|\hat{H}| \chi_{m_{2} n_{2}}\right\rangle$ and the overlap matrix elements $\left\langle\chi_{m_{1} n_{1}} \mid \chi_{m_{2} n_{2}}\right\rangle$. Here, $\chi_{m n}$ is the $n$th atomic orbital of the $m$ th atom. The Hamilton operator contains the kinetic-energy operator as well as the potential. The latter is approximated as a superposition of the potentials of the isolated atoms,

$$
V(\vec{r})=\sum_{m} V_{m}\left(\left|\vec{r}-\vec{R}_{m}\right|\right)
$$

and subsequently we assume that the matrix element $\left\langle\chi_{m_{1} n_{1}}\left|V_{m}\right| \chi_{m_{2} n_{2}}\right\rangle$ vanishes unless at least one of the atoms $m_{1}$ and $m_{2}$ equals $m$. Finally, the pair potentials $U_{m_{1}, m_{2}}$ are obtained by requiring that the total-energy curves from parameter-free density-functional calculations on the diatomics are accurately reproduced.

\section{$2.2 \quad$ Structure determinations}

We used several different methods in determining the structures of the clusters. In the Gaussian03 calculations the clusters were so small that it was possible to determine the structures of the lowest total energy simply through searching in the structure space. In the jellium calculations there is per construction no structure and the system has a spherical symmetry.

In the EAM calculations we optimized the structure using our own Aufbau/Abbau method [38, 39, 40]. The method is based on simulating experimental conditions, where clusters grow by 
adding atom by atom to a core. By repeating this process very many times and in parallel also removing atoms from larger clusters, we can identify the structures of the lowest total energy.

Finally, in the DFTB calculations we used two different approaches. In one approach the structures of the EAM calculations were used as input for a local relaxation, i.e., only the nearest local-total-energy minimum was identified. In another set of calculations, we optimized the structures using the so-called genetic algorithms [41, 42, 43]. Here, from a set of structures we generate new ones through cutting and pasting the original ones. Out of the total set of old and new clusters those with the lowest total energies are kept, and this process is repeated until the lowest total energy is unchanged for a large number of generations.

\section{Results}

The smallest possible cluster is the $\mathrm{Au}_{2}$ molecule. For this we show in Table 1 the calculated bond length and binding energy from the different methods in comparison with experimental values. Notice that the DFTB method has been parameterized to reproduce results from parameter-free density-functional calculations on precisely the dimer and is, therefore, for the dimer accurate.

Table 1: A comparison between the experimental and the calculated bond length and binding energy of the $\mathrm{Au}$ dimer obtained with the parameter-free density-functional calculations and the EAM method.

\begin{tabular}{lcc}
\hline $\mathrm{Au}_{2}$ & $\mathrm{R}_{e}, \AA$ & $\mathrm{E}_{b}, \mathrm{eV}$ \\
\hline $\mathrm{DFT}$ & 2.55 & 2.22 \\
$\mathrm{EAM}$ & 2.40 & 2.29 \\
$E X P$ & 2.47 & 2.29 \\
\hline
\end{tabular}

Next we show in Fig. [1 the structures of $\mathrm{Au}_{N}$ clusters with $4 \leq N \leq 8$ as obtained with the DFT calculations, the DFTB method, and the EAM method. The results for the trimer correspond to an obtuse triangle in the DFT calculations, and an equilateral triangle for the EAM and the DFTB methods. The figure clearly illustrates the aspects we have discussed above, i.e., the optimized structures of $\mathrm{Au}$ clusters result from a subtle interplay between geometric and electronic effects. Thus, in the DFT calculations all clusters are planar, whereas in the EAM calculations they are all three-dimensional (3D). Moreover, it turns out that also relativistic effects are important. Including all relativistic effects (i.e., also spin-orbit couplings) all gold clusters form planar structures at least up to $N=13$ (see Ref. [5]). If the spin-orbit coupling is neglected, one obtains 3D global minima already at $N=4$.

The jellium model excludes packing effects and treats exclusively electronic-shell effects. With $r_{s}$ being the electron-gas parameter of the system of interest (i.e., the radius of a sphere containing one electron) it is well-known [44 that particularly stable clusters (magic numbers) occur for regularly spaced spherical clusters whose radius differ by

$$
\Delta R=0.603 r_{s}
$$

for not too small clusters. For even smaller clusters one finds magic numbers for clusters containing $2,8,18,20,34,58,92,132,138,186,254,338, \ldots$ electrons (see, e.g., 45).

In Fig. 2 we show results from the jellium calculations on Au clusters, where it is assumed that each atom contributes with 11 electrons. Thus, the above-mentioned magic numbers are not reached for the gold clusters. Nevertheless, the total energy per atom shows a very regular behaviour that actually can be related to Eq. (5). By comparing the total energy with the orbital 

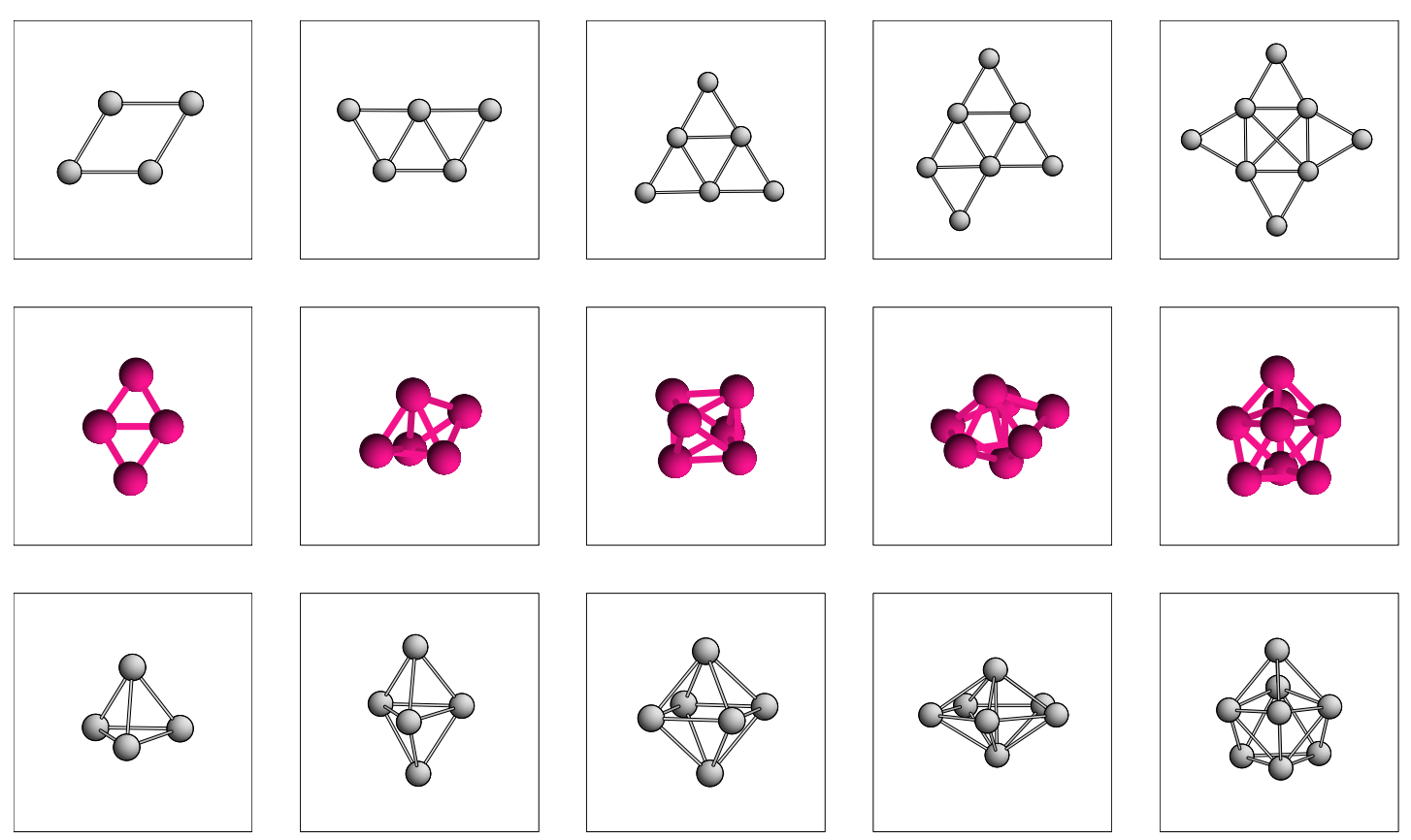

Figure 1: The optimized structures for $\mathrm{Au}_{N}, 4 \leq N \leq 8$ from (upper row) the DFT calculations, (middle row) the DFTB calculations, and (lower row) the EAM calculations.

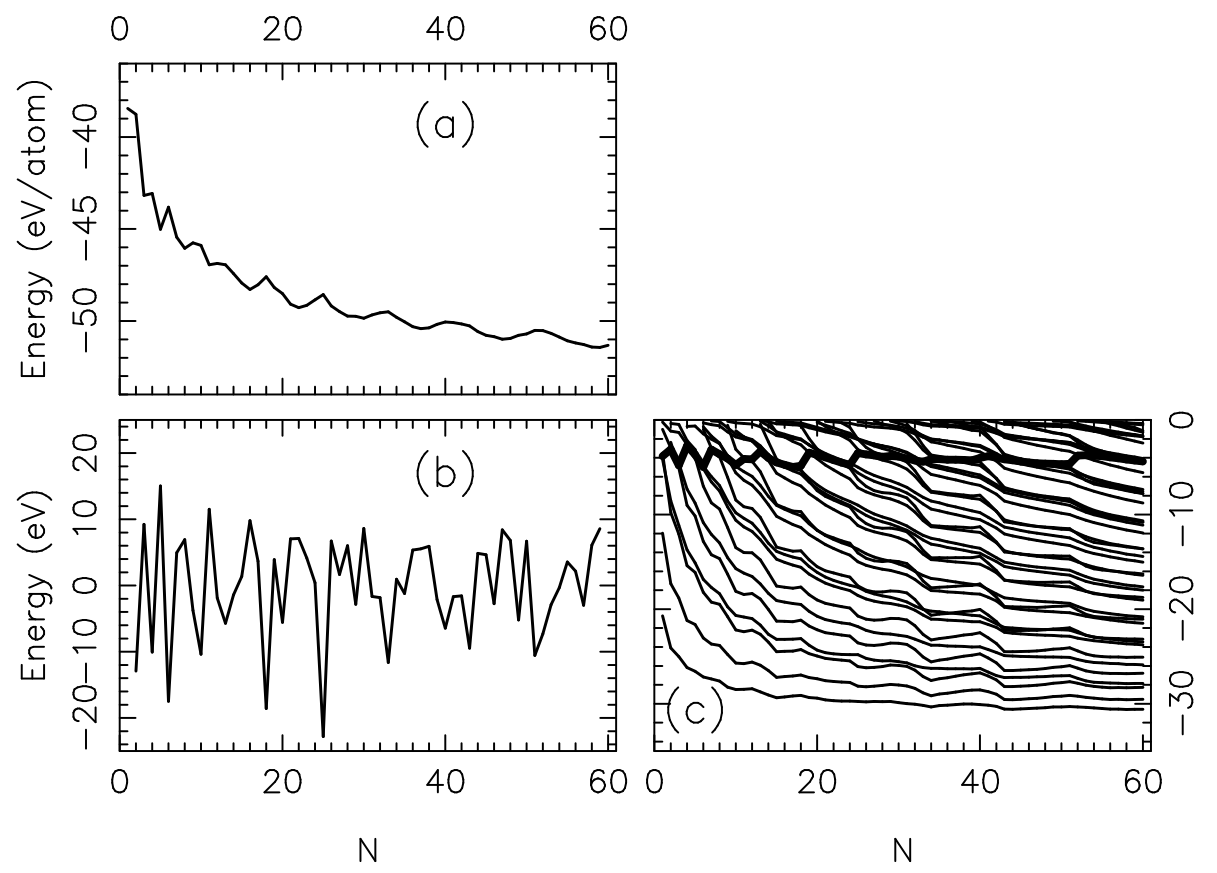

Figure 2: Results from the jellium calculations on $\mathrm{Au}_{N}$ clusters with $1 \leq N \leq 60$. The three panels show (a) total energy per atom, (b) the stability function, and (c) the orbital energies (thin curves) and the Fermi energy (thick curve) as functions of $N$. 
energies (also shown) we see that the local minima correspond to structures where the Fermi level makes a jump, i.e., where new electronic shells are being filled.

In order to identify the particularly stable clusters we introduce the stability function,

$$
\Delta_{2} E(N)=E_{\text {tot }}(N+1)+E_{\text {tot }}(N-1)-2 E_{\text {tot }}(N)
$$

where $E_{\text {tot }}(K)$ is the total energy of the $\mathrm{Au}_{K}$ system. $\Delta_{2} E(N)$ has local maxima when $A u_{N}$ is particularly stable, i.e., when $E_{\text {tot }}(N)$ is particularly low compared with $E_{\text {tot }}(N-1)$ and $E_{\text {tot }}(N+$ 1). This function possesses a number of maxima, as seen in the figure, i.e., for $N=3,8,11,16$, $21,22,26,28,30,36,37,38,47,48,50,58$, and 59 .

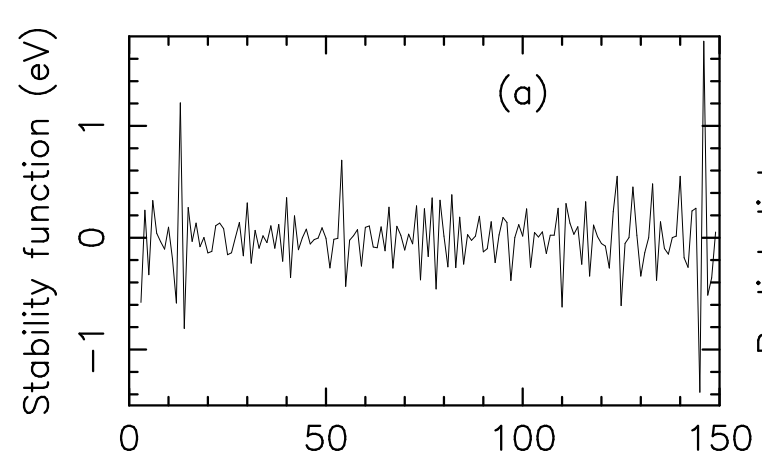

$\mathrm{N}$

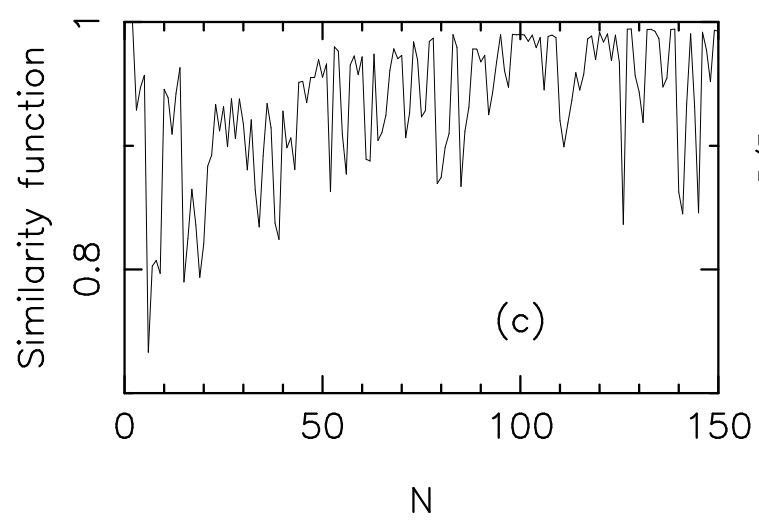

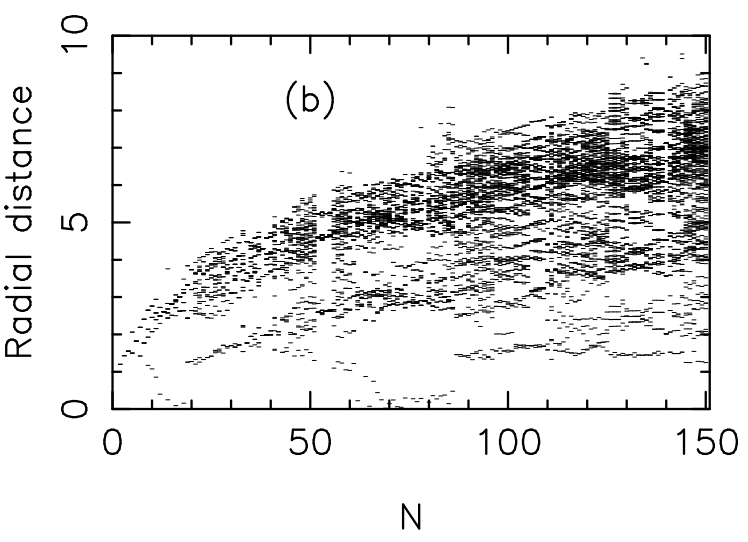

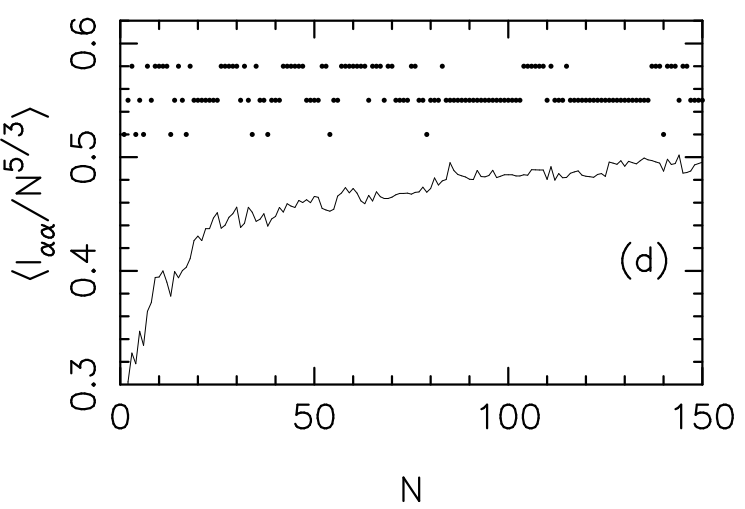

Figure 3: Properties of $\mathrm{Au}_{N}$ clusters from the EAM calculations. The four panels show (a) the stability function, (b) the radial distribution of atoms, (c) the similarity function, and (d) the shape-analysis parameters, respectively. Lengths and energies are given in $\AA$ and $\mathrm{eV}$, respectively. In (d) the upper rows show whether the clusters have an overall spherical shape (lowest row), an overall cigar-like shape (middle row), or an overall lens-like shape (upper row).

We shall now compare this purely electronic description of the clusters with those obtained using the other approaches for $\mathrm{Au}_{N}$. Fig. [3 shows various properties from the EAM calculations on $\mathrm{Au}_{N}$ clusters with $N$ up to 150 . Here, we used our Aufbau/Abbau method in optimizing the structure. The stability function, Fig. 3(a), has pronounced peaks at $N=13,54$, and 146, where the structure 


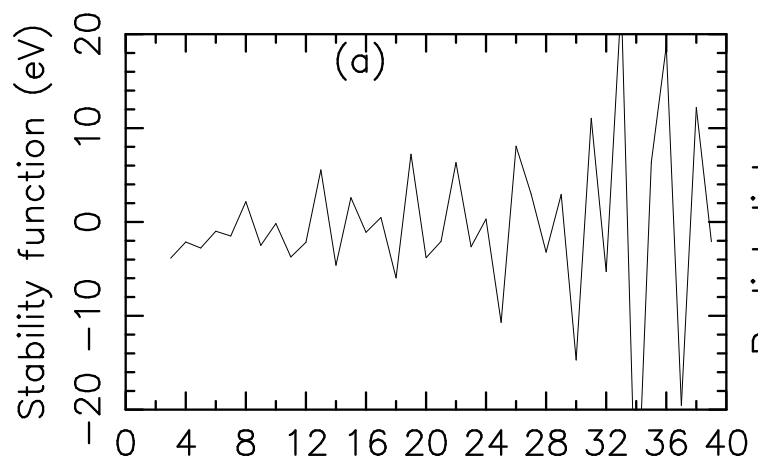

$\mathrm{N}$

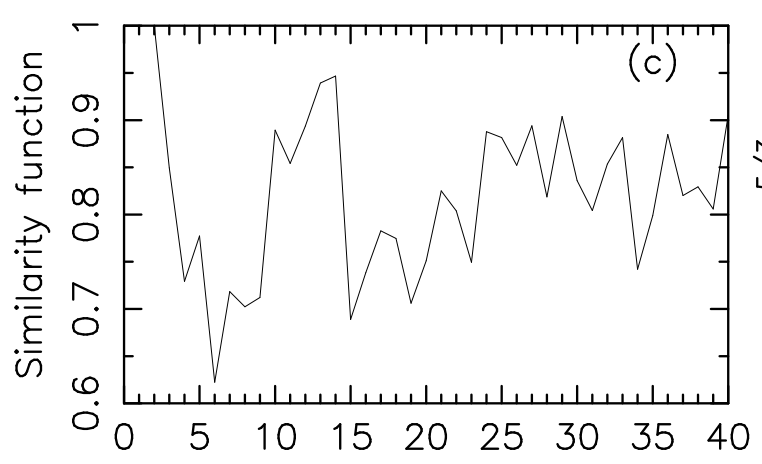

$\mathrm{N}$
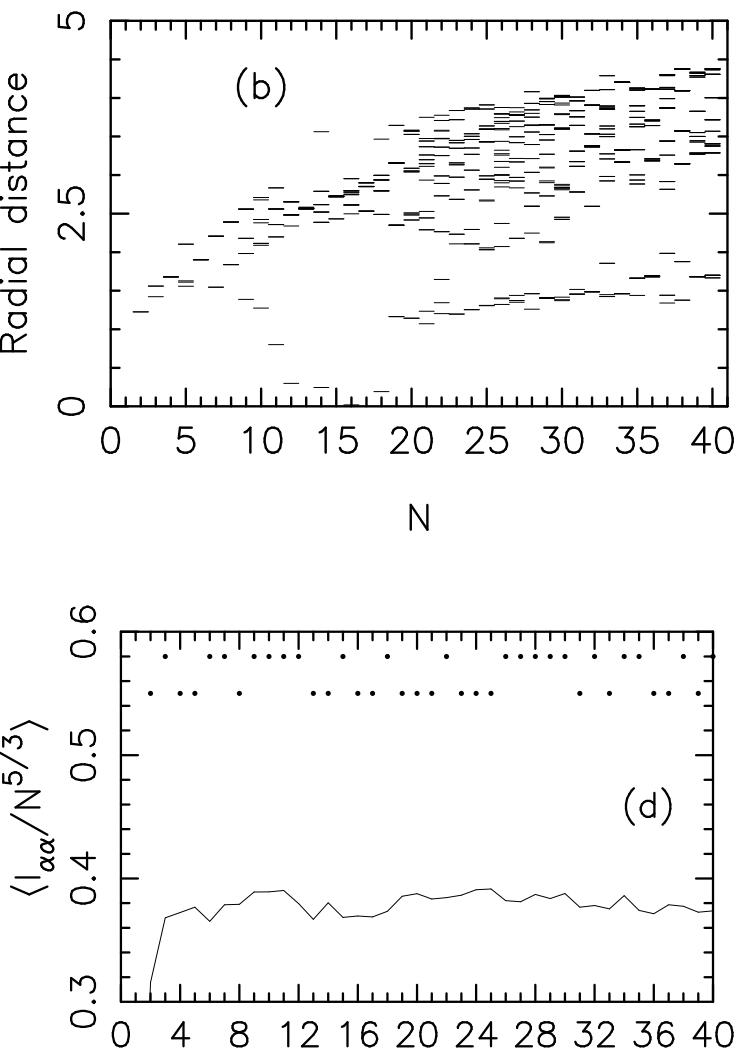

$\mathrm{N}$

Figure 4: As Fig. 3 but from the DFTB calculations with the structures of the EAM calculations.

corresponds to an icosahedron for the first two and a decahedron for the last (actually, for $N=55$ we do not find a icosahedron, whereas the structure of $N=54$ is the 55 -atomic icosahedron without the central atom).

Further information on the structure is obtained from the radial distances of the atoms, defined as follows. First, we define the center of the $\mathrm{Au}_{N}$ cluster,

$$
\vec{R}_{0}=\frac{1}{N} \sum_{i=1}^{N} \vec{R}_{i}
$$

and, subsequently, we define for each atom its radial distance

$$
r_{i}=\left|\vec{R}_{i}-\vec{R}_{0}\right|
$$

In Fig. B(b) we show the radial distances for all atoms and all cluster sizes. Each small line shows that at least one atom for the given value of $N$ has exactly that radial distance. The figure shows that somewhere around $N=10$ a second shell of atoms is being built up, with a central atom for $N=13$. Around $N=54$, there are only few values of the radial distance, i.e., the clusters have a high symmetry. Around $N=75$ we see that a third atomic shell is being formed. 


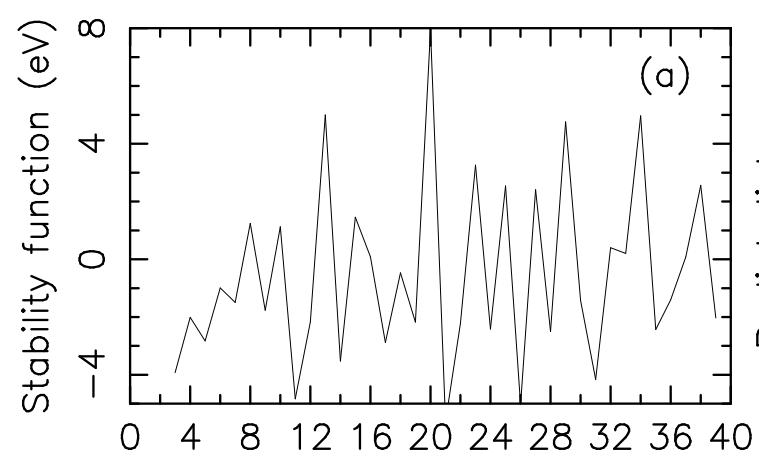

$\mathrm{N}$

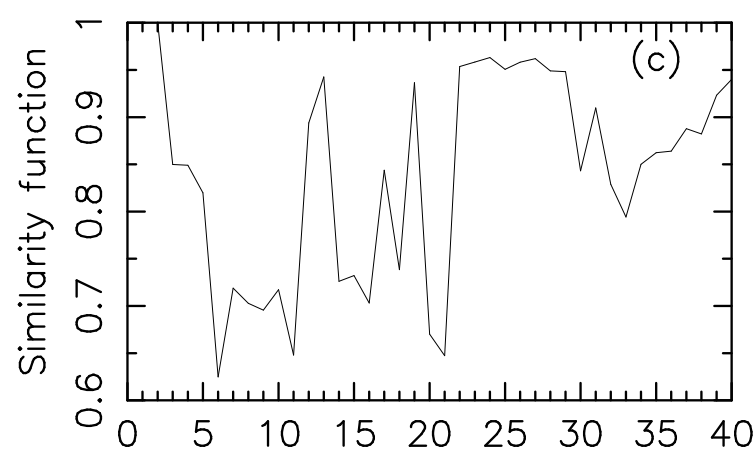

$\mathrm{N}$
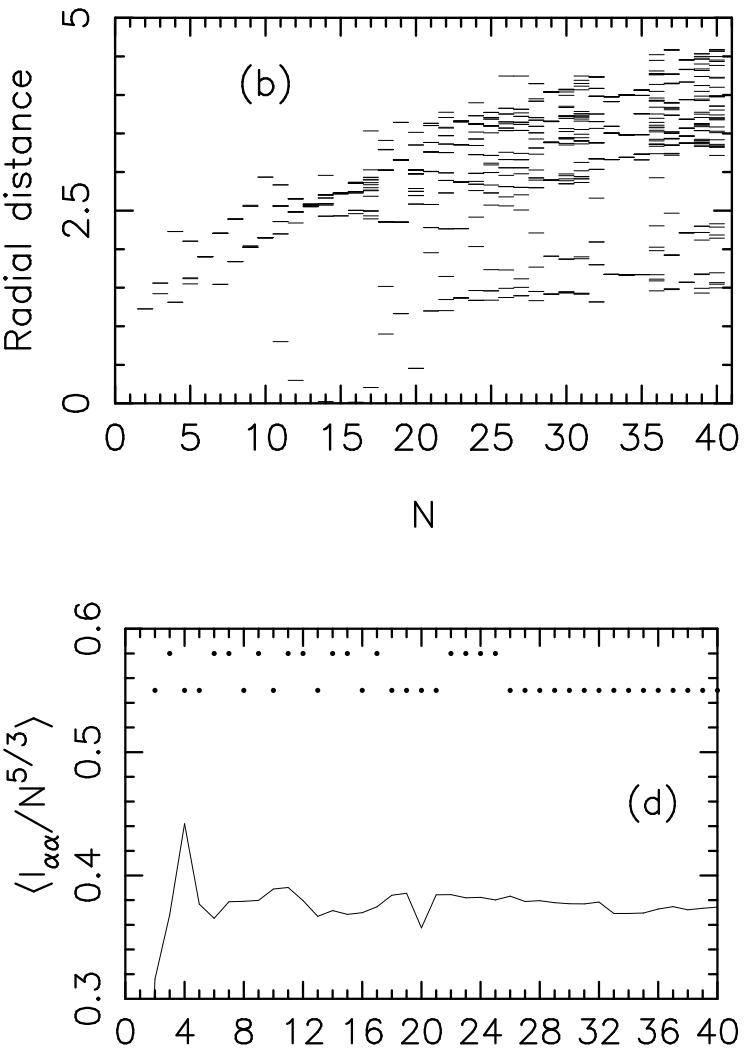

$\mathrm{N}$

Figure 5: As Fig. [3] but from the DFTB calculations with the genetic-algorithm optimization of the structures.

We have earlier found [40] that it was useful to monitor the structural development of the isomer with the lowest total energy through the so-called similarity functions. We shall study how clusters grow and, in particular, if the cluster with $N$ atoms can be derived from the one with $N-1$ atoms simply by adding one extra atom. In order to quantify this relation we consider first the structure with the lowest total energy for the $(N-1)$-atom cluster. For this we calculate and sort all interatomic distances, $d_{i}, i=1,2, \cdots, \frac{N(N-1)}{2}$. Subsequently we consider each of the $N$ fragments of the $N$-cluster that can be obtained by removing one of the atoms and keeping the rest at their positions. For each of those we also calculate and sort all interatomic distances $d_{i}^{\prime}$, and calculate, subsequently,

$$
q=\left[\frac{2}{N(N-1)} \sum_{i=1}^{N(N-1) / 2}\left(d_{i}-d_{i}^{\prime}\right)^{2}\right]^{1 / 2} .
$$

Among the $N$ different values of $q$ we choose the smallest one, $q_{\min }$, and calculate the similarity function

$$
S=\frac{1}{1+q_{\min } / u_{l}}
$$


$\left(u_{l}=1 \AA\right)$ which approaches 1 if the $\mathrm{Au}_{N}$ cluster is very similar to the $\mathrm{Au}_{N-1}$ cluster plus an extra atom. This function is shown in Fig. 3(c). We see that the structural development is very irregular over the whole range of $N$ that we have considered here, with, however, some smaller intervals where $S$ is relatively large, for instance for $N$ slightly above 20 .

Finally, we shall consider the overall shape of the clusters. As we showed in our earlier report on $\mathrm{Ni}$ clusters [40, it is convenient to study the $3 \times 3$ matrix containing the elements

$$
I_{s t}=\frac{1}{u_{l}^{2}} \sum_{n=1}^{N}\left(R_{n, s}-R_{0, s}\right)\left(R_{n, t}-R_{0, t}\right)
$$

with $s$ and $t$ being $x, y$, and $z$. The three eigenvalues of this matrix, $I_{\alpha \alpha}$, can be used in separating the clusters into being overall spherical (all eigenvalues are identical), more cigar-like shaped (one eigenvalue is large, the other two are small), or more lens-shaped (two large and one small eigenvalue). The average of the three eigenvalues, $\left\langle I_{\alpha \alpha}\right\rangle$, is a measure of the overall extension of the cluster. For a homogeneous sphere with $N$ atoms, the eigenvalues scale like $N^{5 / 3}$. Hence, we show in Fig. 3(d) quantities related to $I_{\alpha \alpha}$ but scaled by $N^{-5 / 3}$. In this figure we also mark the overall shape of the clusters through the upper points with the lowest row meaning spherical, the middle row meaning cigar-shaped, and the upper row meaning lens-shaped clusters. Some clusters with an overall spherical shape can be recognized, that, simultaneously, are clusters of particularly high stability according to Fig. [3(a).

By comparing with the results of the jellium calculations, we see that the EAM method predicts a completely different set of particularly stable clusters. Moreover, compared with the results of the parameter-free density-functional calculations, the EAM method tends to produce more compact clusters.

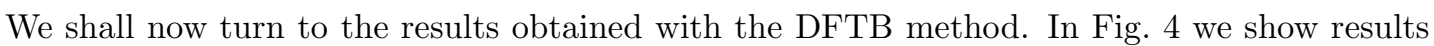
similar to those of Fig. 3 but obtained by letting the clusters of the EAM calculations relax locally using the DFTB method, whereas we in Fig. 5 show the results for the DFTB calculations where the structure was optimized with the genetic algorithms.

The structural information from all three sets of calculations is very similar. The radial distances show in all cases that a second atomic shell is being constructed starting from slightly above 10 atoms. First above $N=40$ a third atomic shell is formed [see Fig. 3(c)]. Moreover, the similarity function shows that clusters in a narrow window just above $N=10$ as well as in a wider window above $N=20$ resemble each other, independently of the theoretical approach. There are, however, some differences in the overall shape, as seen in the shape-analysis parameters. In the EAM calculations several roughly spherical clusters with $N \leq 40$ are found, whereas the symmetry is broken when including electronic effects with the DFTB method, so that in these calculations no cluster is found to have an overall spherical structure. Moreover, the structures of the DFTB calculations are overall slightly more compact than those of the EAM calculations.

On the other hand, the stability function of Fig. 囷a) shows a much more irregular behaviour than the one of Fig. B(a), although the structures are very similar. This emphasizes that electronic effects indeed are important. By comparing with Fig. 囵(a) we see that through further relaxations the stability function becomes less irregular, although its variation (over a range of roughly 12 $\mathrm{eV}$ ) is significantly larger than the range of the stability function in Fig. 3 (a) (roughly $2 \mathrm{eV}$ ). It is interesting to observe that in the jellium calculations (where there is per construction no structural relaxation) the stability function spans a range of roughly $35 \mathrm{eV}$, which is comparable to the range we see in Fig. \&(a). This means that electronic effects are very important for the stability of the clusters, but also that through structural relaxations the role of the electronic effects can be somewhat reduced.

Finally, we see in Fig. 1 that the DFTB calculations lead to essentially the same (type of) structures as the EAM calculations, with $N=4$ being the only significant exception. Here, the 
DFTB calculations predict a planar structure (as is the case for the relativistic DFT calculations), whereas for larger values of $N$, non-planar structures result in all our calculations except for the relativistic DFT calculations.

\section{Conclusions}

In this work we have discussed the interplay of electronic and packing effects in clusters. We have used $\mathrm{Au}_{N}$ clusters as a prototype in order to illustrate the effects of different types of approximations on the description of the interatomic interactions. We have, moreover, demonstrated how carefully chosen descriptors can be constructed that clearly grasp the essential outcomes of the calculations. Moreover, we hope also to have demonstrated the complexities related to unbiased, accurate calculations of the properties of not-too-small clusters.

Our results indicate the existence of a tendency for simple potentials that do not directly include effects due to electronic orbitals (i.e., due to directed chemical bonds) to prefer closed packed structures. Including the electronic orbitals the structures may become less symmetric, and when increasing the accuracy of the treatment of the electronic orbitals and, simultaneously, the structure, the geometric arrangement of the atoms becomes less and less closed packed. Moreover, the electronic effects lead to a much stronger variation in stability as a function of size.

We have in this presentation focused on $\mathrm{Au}_{N}$ clusters. These clusters are among the most studied ones and are simultaneously very difficult to treat theoretically (as discussed in the introduction), which was the motivation for the present work. Nevertheless, we believe that most of our conclusions are valid also for other types of metal clusters, thus emphasizing the importance to perform different types of calculations with different types of (approximate) descriptions of the interatomic interactions before making finite conclusions about the properties of the clusters of a specific element.

\section{Acknowledgment}

We gratefully acknowledge Fonds der Chemischen Industrie for very generous support. This work was supported by the SFB 277 of the University of Saarland and by the German Research Council (DFG) through project Sp439/14-1.

\section{References}

[1] P. Pyykkö, Angew. Chem. Int. Ed. 43, 4412 (2004).

[2] M. Dorogi, J. Gomez, R. Osifichin, R. P. Andres, and R. Reifenberger, Phys. Rev. B 52, 9071 (1995).

[3] R. L. Whetten, M. N. Shafigullin, J. T. Khoury, T. G. Schaaff, I. Vezmar, M. M. Alvarez, and A. Wilkinson, Acc. Chem. Res. 32, 397 (1999).

[4] A. Sanchez, S. Abbet, W. D. Schneider, H. Häkkinen, R. N. Barnett, and U. Landman, J. Phys. Chem. A 103, 9573 (1999).

[5] H. Häkkinen, B. Yoon, U. Landman, X. Li, H.-J. Zhai, and L.-S. Wang, J. Phys. Chem. A 107, 6168 (2003).

[6] S. Gilb, P. Weis, F. Furche, R. Ahlrichs, and M. M. Kappes, J. Chem. Phys 116, 4094 (2002). 
[7] M. Neumaier, F. Weigend, and O. Hampe, J. Chem. Phys. 122, 104702 (2005).

[8] G. Bravo-Pérez, I. L. Garzón, and O. Novaro, J. Mol. Str. (Theochem) 493, 225 (1999).

[9] H. Grönbeck and W. Andreoni, Chem. Phys. 262, 1 (2000).

[10] H. Häkkinen and U. Landman, Phys. Rev. B 62, 2287 (2000).

[11] J. Wang, G. Wang, and J. Zhao, Phys. Rev. B 66, 035418 (2002).

[12] A. V. Walker, J. Chem. Phys. 122, 094310 (2005).

[13] F. Remacle and E. S. Kryachko, J. Chem. Phys. 122, 044304 (2005).

[14] J. P. K. Doye and D. J. Wales, New J. Chem. 733 (1998).

[15] N. T. Wilson and R. L. Johnston, Eur. Phys. J. D 12, 161 (2000).

[16] O. D. Häberlen, S. C. Chung, M. Stener, and N. Rösch, J. Chem. Phys. 106, 5189 (1997).

[17] C. L. Cleveland, U. Landman, T. G. Schaaff, M. N. Shafigullin, P. W. Stephens, and R. L. Whetten, Phys. Rev. Lett. 79, 1873 (1997).

[18] R. N. Barnett, C. L. Cleveland, H. Häkkinen, W. D. Luedtke, C. Yannouleas, and U. Landman, Eur. Phys. J. D 9, 95 (1999).

[19] C. L. Cleveland, U. Landman, M. N. Shafigullin, P. W. Stephens, and R. L. Whetten, Z. Phys. D 40, 503 (1997).

[20] I. L. Garzón and A. Posada-Amarillas, Phys. Rev. B 54, 11796 (1996).

[21] I. L. Garzón, K. Michaelian, M. R. Beltrán, A. Posada-Amarillas, P. Ordejón, E. Artacho, D. Sánchez-Portal, and J. M. Soler, Phys. Rev. Lett. 81, 1600 (1998).

[22] I. L. Garzón, K. Michaelian, M. R. Beltrán, A. Posada-Amarillas, P. Ordejón, E. Artacho, D. Sánchez-Portal, and J. M. Soler, Eur. Phys. J. D 9, 211 (1999).

[23] K. Michaelian, N. Rendón, and I. L. Garzón, Phys. Rev. B 60, 2000 (1999).

[24] T. X. Li, S. Y. Yin, Y. L. Ji, B. L. Wang, G. H. Wang, and J. J. Zhao, Phys. Lett. A 267, $403(2000)$.

[25] S. Darby, T. V. Mortimer-Jones, R. L. Johnston, and C. Roberts, J. Chem. Phys. 116, 1536 (2002).

[26] F. Baletto and R. Ferrando, Rev. Mod. Phys. 77, 371 (2005).

[27] V. G. Grigoryan, D. Alamanova, and M. Springborg, Eur. Phys. J. D (in press).

[28] M. J. Frisch, G. W. Trucks, H. B. Schlegel, G. E. Scuseria, M. A. Robb, J. R. Cheeseman, J. A. Montgomery Jr., T. Vreven, K. N. Kudin, J. C. Burant, J. M. Millam, S. S. Iyengar, j. Tomasi, V. Barone, B. Mennucci, M. Cossi, G. Scalmani, N. Rega, G. A. Petersson, H. Nakatsuji, M. Hada, M. Ehara, K. Toyota, R. Fukuda, J. Hasegawa, M. Ishida, T. Nakajima, Y. Honda, O. Kitao, H. Nakai, M. Klene, X. Li, J. E. Knox, H. P. Hratchian, J. B. Cross, C. Adamo, J. Jaramillo, R. Gomberts, R. E. Strattmann, O. Yazyev, A. J. Austin, R. Cammi, C. Pomelli, J. W. Ochterski, P. Y. Ayala, K. Morokuma, G. A. Voth, P. Salvador, J. J. Dannenberg, V. G. Zakrzewski, S. Dapprich, A. D. Daniels, M. C. Strain, O. Farkas, D. K. Malick, A. D. 
Rabuck, K. Raghavachari, J. B. Foresman, J. V. Ortiz, Q. Cui, A. G. Baboul, S. Clifford, J. Cioslowski, B. B. Stefanov, G. Liu, A. Liashenko, P. Piskorz, I. Komaromi, R. L. Martin, D. J. Fox, T. Keith, M. A. Al-Laham, C. Y. Peng, A. Nanayakkara, M. Challacombe, P. M. W. Gill, B. Johnson, W. Chen, M. W. Wong, C. Gonzalez, and J. A. Pople, Gaussian03, revision C.02, Gaussian Inc., Wallingford, CT, 2004.

[29] J. P. Perdew, K. Burke, and M. Ernzerhof, Phys. Rev. Lett. 77, 3865 (1996).

[30] J. P. Perdew, K. Burke, and M. Ernzerhof, Phys. Rev. Lett. 78, 1396 (1997).

[31] W. A. de Heer, Rev. Mod. Phys. 65, 611 (1993).

[32] M. Brack, Rev. Mod. Phys. 65, 677 (1993).

[33] A. F. Voter and S. P. Chen, in Characterization of Defects in Materials, edited by R. W. Siegal, J. R. Weertman, and R. Sinclair, MRS Symposia Proceedings No. 82 (Materials Research Society, Pittsburgh, 1987), p. 175.

[34] A. Voter, Los Alamos Unclassified Technical Report No LA-UR 93-3901 (1993).

[35] A. F. Voter, in Intermetallic Compounds, edited by J. H. Westbrook and R. L. Fleischer (John Wiley and Sons, Ltd, 1995), Vol. 1, p. 77.

[36] G. Seifert and R. Schmidt, New J. Chem. 16, 1145 (1992).

[37] G. Seifert, D. Porezag, and Th. Frauenheim, Int. J. Quant. Chem 58, 185 (1996).

[38] V. G. Grigoryan and M. Springborg, Phys. Chem. Chem. Phys. 3, 5125 (2001).

[39] V. G. Grigoryan and M. Springborg, Chem. Phys. Lett. 375, 219 (2003).

[40] V. G. Grigoryan and M. Springborg, Phys. Rev. B 70, 205415 (2004).

[41] J.-O. Joswig, M. Springborg, and G. Seifert, Phys. Chem. Chem. Phys. 3, 5130 (2001).

[42] J.-O. Joswig and M. Springborg, Phys. Rev. B 68, 085408 (2003).

[43] Y. Dong and M. Springborg, in Proceedings of 3rd International Conference "Computational Modeling and Simulation of Materials", Ed. P. Vincenzini et al., Techna Group Publishers, p. 167 (2004).

[44] R. Balian and C. Bloch, Ann. Phys. New York 69, 76 (1972).

[45] M. Springborg, J. Phys. Cond. Matt. 11, 1 (1999). 